\title{
An Approach to Data Reduction and Decision Making Problem by Hybridization of Fuzzy Soft Set and Graph Theory
}

\author{
Omdutt Sharma \\ Research Scholar \\ Department of Statistics, \\ M.D. University, Rohtak
}

\author{
Prince Goyal, PhD \\ Tutor cum Statistician \\ Department of Community \\ Medicine, Dr. YSPG Medical \\ College, Nahan, Himachal
}

\author{
Priti Gupta \\ Prof. and Head \\ Department of Statistics, \\ M.D. University, Rohtak
}

\begin{abstract}
To deal with the uncertainties present in real life situations both fuzzy set and soft set approach are concerned. But these approaches have some challenges, to solve related dilemma a hybridization approach is operated known as fuzzy soft set approach, which is the hybridization of fuzzy sets and soft set theory. Graph theory is also a significant tactic to solve some existent problem graphically. In this paper, a brief study of fuzzy soft sets and graph theory are done. Here a definition of fuzzy soft graph is defined and give a method to solve decision making problem and data reduction problem by using fuzzy soft set definition defined by us. At last discuss an application of decision making and data reduction problem by using fuzzy soft sets and graph theory.
\end{abstract}

\section{General Terms}

Feature Selection and Decision Making Method

\section{Keywords}

Fuzzy set, soft set, fuzzy soft set, graph theory, soft graph, fuzzy soft graph, feature selection, decision making.

\section{INTRODUCTION}

Zadeh [1] proposed a theory which is beneficial to handle the problems related to uncertainty and vagueness in real life, which is non-probabilistic in nature. Which is knew as fuzzy set theory. Due to its potential for managing uncertainty, fuzzy set has attains huge sensation. Like all other theories, this theory has a drawback to allocate membership value. Due to its difficulty to allocate the membership function, it is not simply applied for some tricky challenges to economics, engineering, environmental science, social science and other real life situations. To deal with these types of dilemmas, a significant theory is insinuated by Molodtsov [2], known as soft sets theory, which is a new mathematical tool for trading with those uncertainties which are not conducted by traditional mathematical tool. Basically, a soft set is a parameterized classification of the object of the universe. In his work, he discussed several applications of soft sets in various area like integration, decision making etc. Since after his proposal, works on soft set theory is developing rapidly. A theoretical study on the theory of soft sets and several algebraic operations on soft sets were defined by Maji et al. [5]. Maji et al. [4] also solve some decision making problems by using soft sets. Maji et al. [3] also introduced the concept of fuzzy soft set, a more generalized concept, which is a combination of fuzzy set and soft set and studied its properties. By using this definition of fuzzy soft sets many interesting application of soft set theory has been expanded by some researchers. Roy and Maji [6] discussed some application of fuzzy soft sets. Zou and Xio [13] introduced the soft set and fuzzy soft set into the incomplete environment. Yang et al. [14] presented the combination of interval-valued fuzzy set and soft set. Chen et al. [7] proposed parameterization reduction of soft sets and then Kong et al. $[15,16]$ defined the normal parameter reduction in the soft sets and decision making in fuzzy soft sets and showed that Roy and Maji [6] algorithm is not convenient in general cases.

In mathematics, some mathematical structures are used to model pair-wise relations between objects, these structures are known as graphs. The theory in which we study about graph is known as graph theory. The notion of graph theory was commenced by Euler (1736). To handle combinatorial problems of various fields such as geometry, algebra, number theory, topology, operation research, optimization and computer science, etc, this theory is a significant tool. Various researchers combine graph theory of other theory like fuzzy theory by Rosenfeld [9], soft set theory by Thumbakara and George [10], fuzzy soft set theory by Mohinta and Samanta, [11]. Thomas and Arockiarani [12] discuss an application of soft graph in decision making. The rest of paper is arranged as:

In section second theoretical background some detail or related concept and definition are discussed which are used in this paper. In section third proposed method in which a definition of fuzzy soft graph are defined and give the step wise procedure for decision making and feature selection problems [8]. In next section an application related to feature selection and decision making problems are discussed. At last conclude the paper.

\section{THEORETICAL BACKGROUND}

In this section some related concepts and definition related to this work are discussed.

\subsection{Fuzzy Set}

In mathematics, fuzzy sets are those sets whose elements have degree of membership. As an extension of the classical notion of a set Lotfi A. Zadeh [1] pioneered fuzzy sets.

\section{Definition 2.1}

A fuzzy set is a pair $(U, m)$ where $U$ is a set and $m: U \rightarrow$ $[0,1]$ for each $y \in U$, the value $m(y)$ is called the grade of membership of $y$ in $(U, m)$. For a finite set $U=$ $\left\{y_{1}, y_{2}, \ldots, y_{n}\right\}$, the fuzzy set $(U, m)$ is often denoted by $\left\{m\left(y_{1}\right) /\left(y_{1}\right), \ldots \ldots . . m\left(y_{n}\right) /\left(y_{n}\right)\right\}$.

\section{Membership Function:}

In the fuzzy set $R$ the membership function, $m_{R}(y)$ exemplifies the membership of the element $y$ of the base set $Y$, where for $m_{R}(y)$ a large class of function can be taken. For the fuzzy set $R$, the grade of membership $m_{R}\left(y_{0}\right)$ of a 
membership function $m_{R}(y)$ express for the special element $y=y_{0}$. This value is in the unit interval $[0,1]$.

\subsection{Soft Set}

Soft set theory is an overview of fuzzy set theory that was suggested by Molodtsov [2] to manage uncertainty of a parametric approach. Due to the lack of any limitations on the approximate descriptions, soft set theory is very convenient and easily suitable for execution. In this theory, we can conduct any parameter that we desire, with the aid of words and sentences, real number, function, mapping and so on.

\section{Definitions 2.2}

Let $Z$ is an initial universe set and $P$ is the set of parameters, than the pair $(\mathcal{H}, P)$ is called a soft set (over $Z$ ) if $\mathcal{H}$ is a mapping of $P$ into the power set of $Z$, that is,

$$
\mathcal{H}: P \rightarrow P(Z) \text {. }
$$

Clearly, for a given universe $Z$ a soft set is a parameterized family of subsets over $Z$. Also for any $p \in P, \mathcal{H}(p)$ is considered as the set of p-approximate element of the soft set $(\mathcal{H}, E)$.

\section{Example 1:}

Let $Z=\left\{m_{1}, m_{2}, \ldots, m_{5}\right\}$, be the sets of motor vehicle under consideration and $P$ be the set of parameters,

$$
\begin{array}{r}
P=\left\{p_{1}=\text { expensive, } p_{2}=\text { good quality, } p_{3}\right. \\
\left.=\text { cheap, } p_{4}=\text { stylish, } p_{5}=\text { latest }\right\} .
\end{array}
$$

Then the soft set $(\mathcal{H}, P)$ express the attractiveness of the mobiles.

$$
(\mathcal{H}, P)=\left\{\begin{array}{c}
\left\{\text { expensive }=m_{1}, m_{2}, m_{5}\right\}, \\
\left\{\text { good quality }=m_{3}, m_{5}\right\}, \\
\left\{\text { cheap }=m_{3}, m_{4}, m_{5}\right\}, \\
\left\{\text { stylish }=m_{1}, m_{2}, \ldots, m_{5}\right\}, \\
\left\{\text { latest }=m_{1}, m_{3}, m_{4}, m_{5}\right\}
\end{array}\right\}
$$

\subsection{Fuzzy Soft Set:}

By hybridization of fuzzy sets and soft sets Maji et al. [3] characterized fuzzy soft sets. They established the notation of fuzzy soft sets, which can be seen as a fuzzy generalization of (classical) soft sets. The definition of fuzzy soft set is followed by an example.

\section{Definition 2.3}

Let $F(Z)$ denote the set of all fuzzy sets of universal set $Z$ and $P$ be the set of parameters. Then a fuzzy soft set $F_{E}$ over $Z$ is a set defined by a function $\Gamma_{E}$ representing a mapping

$$
\Gamma_{E}: \mathrm{P} \rightarrow \mathrm{F}(\mathrm{Z})
$$

Such that $\Gamma_{E}(p)=\emptyset$ if $p \notin E$, where $E \subset P$ and $\Gamma_{E}(p)$ is a fuzzy set over $Z$ for all $p \in P$.

Thus, a fuzzy soft set $F_{E}$ over $Z$ can be represented by the set of ordered pairs

$$
F_{E}=\left\{\left(p, \Gamma_{E}(p)\right): p \in P, \Gamma_{E}(p) \in F(Z)\right\}
$$

A fuzzy soft set is actually a special case of a soft set because it is still a mapping from parameters to a universe. The difference between a soft set and a fuzzy soft set is that in a fuzzy soft set, the universe to be considered is the set of fuzzy subsets of $Z$.

Remark: Note that the set of all fuzzy soft sets over $U$ will be denoted by $\mathrm{FS}(Z)$.

\section{Example 2:}

In example 1 of soft set considered above if $m_{2}$ has stylish then it will not be possible to express it with only the two numbers 0 and 1 . In that case we can characterize it by a membership function instead of the crisp number 0 and 1 that associated with each element a real number in the interval $[0$, 1]. The fuzzy soft set can then be described as

$$
F_{P}=\left\{\begin{array}{l}
F_{P}\left(p_{1}\right)=\left\{\frac{m_{1}}{0.36}, \frac{m_{2}}{0.24}, \frac{m_{3}}{0}, \frac{m_{4}}{0}, \frac{m_{5}}{0.6}\right\}, \\
F_{P}\left(p_{2}\right)=\left\{\frac{m_{1}}{0}, \frac{m_{2}}{0}, \frac{m_{3}}{0.32}, \frac{m_{4}}{0}, \frac{m_{5}}{0.40}\right\}, \\
F_{P}\left(p_{3}\right)=\left\{\frac{m_{1}}{0}, \frac{m_{2}}{0}, \frac{m_{3}}{0.48}, \frac{m_{4}}{0.36}, \frac{m_{5}}{0.6}\right\}, \\
F_{P}\left(p_{4}\right)=\left\{\frac{m_{1}}{0.6}, \frac{m_{2}}{0.4}, \frac{m_{3}}{0.80}, \frac{m_{4}}{0.60}, \frac{m_{5}}{1}\right\}, \\
F_{P}\left(p_{5}\right)=\left\{\frac{m_{1}}{0.48}, \frac{m_{2}}{0}, \frac{m_{3}}{0.64}, \frac{m_{4}}{0.48}, \frac{m_{5}}{0.8}\right\}
\end{array}\right\}
$$

\subsection{Concept of Graph Theory}

A graph $G=(V, E)$ consists of a non-empty set of objects $V$, called vertices and a set $E$ of two element subsets of $V$ called edges. Two vertices $x$ and $y$ are adjacent if $\{x, y\} \in E$. A graph $G=\left(V^{\prime}, E^{\prime}\right)$ is said to be a subgraph of $G=(V, E)$ if $V^{\prime} \subseteq V$ and $E^{\prime} \subseteq E$. For any subset $S$ of the vertex set of the graph $G$, the induced subgraph $S^{\prime}$ is the subgraph of $G$ whose vertex set is $S$ and two vertices are adjacent in $S$ if and only if they are adjacent to G. A graph is complete if every vertex is connected to every other vertex and we denote the complete graph on $n$ vertexes by $K_{n}$.

\section{Definition 2.4.1:}

A graph $G$ is called bipartite if $V(G)$ is the union of two disjoint nonempty subset $V_{1}$ and $V_{2}$ such that every edge of $G$ joins a vertex of $V_{1}$ to a vertex of $V_{2}$. A graph is called a complete bipartite graph if, in addition, every vertex of $V_{1}$ is joined to every vertex of $V_{2}$ by a unique edge.

\subsection{Soft Graph:}

Let $G=(V, E)$ be a simple graph, $A$ any non empty set. Let $R$ any arbitrary relation between elements of $A$ and elements of $V$. That is $R \subseteq A \times V$. A set valued function $F: A \rightarrow P(V)$ can be defined as $F(x)=\{y \in V \mid x R y\}$. The pair $(F, A)$ is a soft set over $V$.

\section{Definition 2.5.1:}

Let $(F, A)$ be a soft set over $V$. Thus $(F, A)$ is said to be a soft graph of $G$ if the subgraph induced by $F(x)$ in $G, F(x)$ is connected subgraph of $G$ for all $x \in A$.

Definition 2.5.2( Akram et al [18]):

A 4-tuple $G=\left(G^{*}, F, K, A\right)$ is called a soft graph if it satisfies the following conditions:

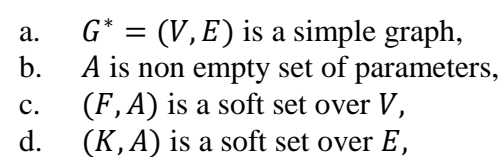

$(F(a), K(a))$ is a sub graph of $G^{*}$ for all $a \in A$.

The sub graph $(F(a), K(a))$ is denoted by $H(a)$ for convenience.

The set of all soft graph of $G^{*}$ is denoted by $S G\left(G^{*}\right)$.

Definition 2.5.3 (Akram et al [18]):

A soft graph $G=\left(G^{*}, F, K, A\right)$ is said to be vertex-induced if 


$$
H(x)=(F(x), K(x))=\langle F(x)\rangle, \forall x \in A .
$$

Definition ( Akram et al [18]):

A soft graph $G=\left(G^{*}, F, K, A\right)$ is said to be edge-induced if

$$
H(x)=(F(x), K(x))=\langle K(x)\rangle, \forall x \in A .
$$

\subsection{Fuzzy Soft Graph:}

Definition 2.6.1 ( Akram et al [17]):

A fuzzy soft graph $\widetilde{G}=\left(G^{*}, \widetilde{F}, \widetilde{K}, A\right)$ is a 4-tuple such that

e. $G^{*}=(V, E)$ is a simple graph,

f. $A$ is non empty set of parameters,

g. $(\tilde{F}, A)$ is a fuzzy soft set over $V$,

h. $(\widetilde{K}, A)$ is a fuzzy soft set over $E$,

i. $\quad(\widetilde{F}(a), \widetilde{K}(a))$ is a fuzzy (sub)graph of $G^{*}$ for all $a \in A$. That is,

$$
\widetilde{K}(a)(x y) \leq \min \{\tilde{F}(a)(x), \tilde{F}(a)(y)\}
$$

$\forall a \in A \& x, y \in V$, the fuzzy graph $(\widetilde{F}(a), \widetilde{K}(a))$ is denoted by $\widetilde{H}(a)$ for convenience.

\section{Definition 2.6.2:}

A fuzzy soft graph $\tilde{G}=\left(G^{*}, \tilde{F}, \widetilde{K}, A\right)$ is said to be vertexinduced if

$$
\begin{gathered}
\widetilde{H}(a)(x)=((\tilde{F}(a)(x), \widetilde{K}(a)(x)))=\langle\tilde{F}(a)(x)\rangle, \forall a \in A \& x \\
\in V .
\end{gathered}
$$

Definition 2.6.3:
A fuzzy soft graph $\tilde{G}=\left(G^{*}, \tilde{F}, \widetilde{K}, A\right)$ is said to be edgeinduced if

$$
\begin{gathered}
\widetilde{H}(a)(x y)=((\tilde{F}(a)(x y), \widetilde{K}(a)(x y)))=\langle\widetilde{K}(a)(x y)\rangle, \forall a \\
\in A \& x, y \in V .
\end{gathered}
$$

\section{PROPOSED METHOD}

Before discussion of this method one more definition related to soft graph is discussed and by using this definition a corresponding definition of fuzzy soft graph is defined. This definition used by Thomas et al [12] in his work related to "decision making via soft graphs"

\section{Definition 3.1:}

Let $\left(F_{A}, E\right)$ be a soft set over $U$. Then a subset $R_{A}$ of $U \times E$, uniquely defined as $R_{A}=\left\{(u, e): e \in A, u \in F_{A}(e)\right\}$, is called a relation form of the soft set $\left(F_{A}, E\right)$.

Let

$U=\left\{u_{1}, u_{2}, u_{3}, \ldots \ldots \ldots, u_{m}\right\}, E=$

$\left\{e_{1}, e_{2}, e_{3}, \ldots \ldots \ldots, e_{n}\right\}$ and $A \subseteq E$. Then $\quad R_{A} \quad$ can $\quad$ be represented as follows:

$R_{A}=\left\{\left(u_{i}, e_{j}\right)\right\}, w \square$ ere $i=1$ to $m$ and $j=1$ to $n$, be the set of points on the graph. Then we can represent a graph and call it a soft graph of the set $\left(F_{A}, E\right)$ over $U$. In other words, a soft set is uniquely represented by its corresponding soft graph.

Fig. 1 Soft Graph

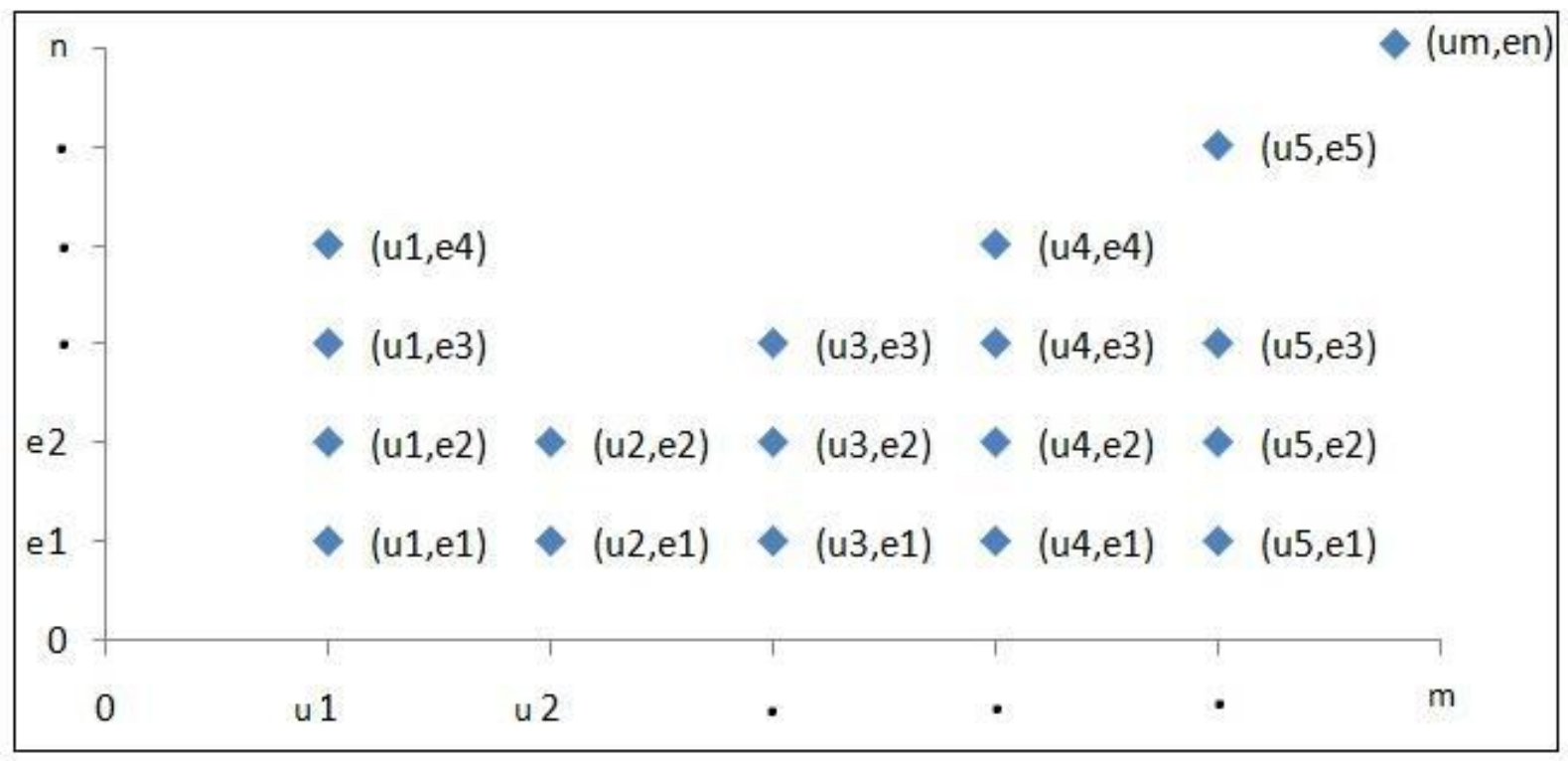

Example 3: The soft graph of example 1 defined in soft set definition is as: 


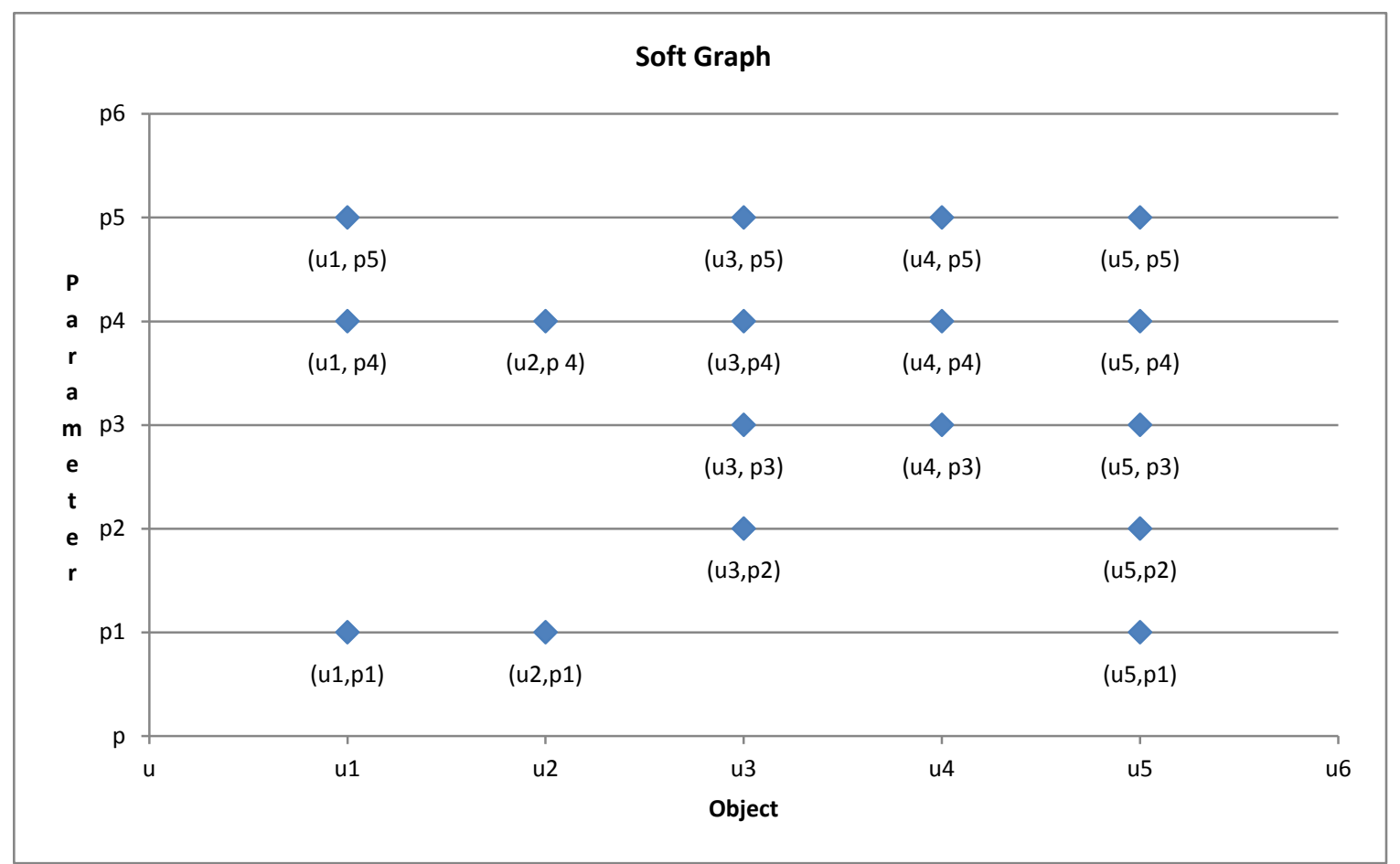

Fig. 2. Soft Graph of Example

Corresponding to above definition we define fuzzy soft graph definition as:

\section{Definition 3.2:}

Let $\mathcal{F}_{E}=\left\{\left(e, \Gamma_{E}(e)\right): e \in P, P \subseteq E, \Gamma_{E}(e) \in F(U)\right\}$ be a fuzzy soft set over universe $\mathrm{U}$. Then a subset $\mathfrak{R}_{P}$ of $U \times E$, uniquely defined as $\mathfrak{R}_{P}=\left\{\left(\Gamma_{E}(e), e\right): e \in P, \Gamma_{E}(e) \in\right.$ $F U$, is called a relation form of the fuzzy soft set $\mathcal{F}_{E}$.

Let $U=\left\{u_{1}, u_{2}, u_{3}, \ldots \ldots \ldots, u_{m}\right\}$,

$E=\left\{e_{1}, e_{2}, e_{3}, \ldots \ldots \ldots, e_{n}\right\}$ and $P \subseteq E$. Then $\mathfrak{R}_{P}$ can be represented as follows:
$\mathfrak{R}_{P}=\left\{\left(\Gamma_{E}\left(e_{j}\right)_{i}, e_{j}\right)\right\}$, where $i=1$ to $m$ and $j=1$ to $n$, be the set of points on the graph. Thus we can represent a graph, and call it fuzzy soft graph of the set $\mathcal{F}_{E}$ over $U$. In other words, a fuzzy soft set is uniquely represented by its corresponding fuzzy soft graph. A fuzzy soft graph shown in fig. 3 below in this figure $\Gamma_{E}\left(e_{j}\right)_{i}$ is represented by $u_{i}$, for $i=1$ to $\mathrm{m}$.

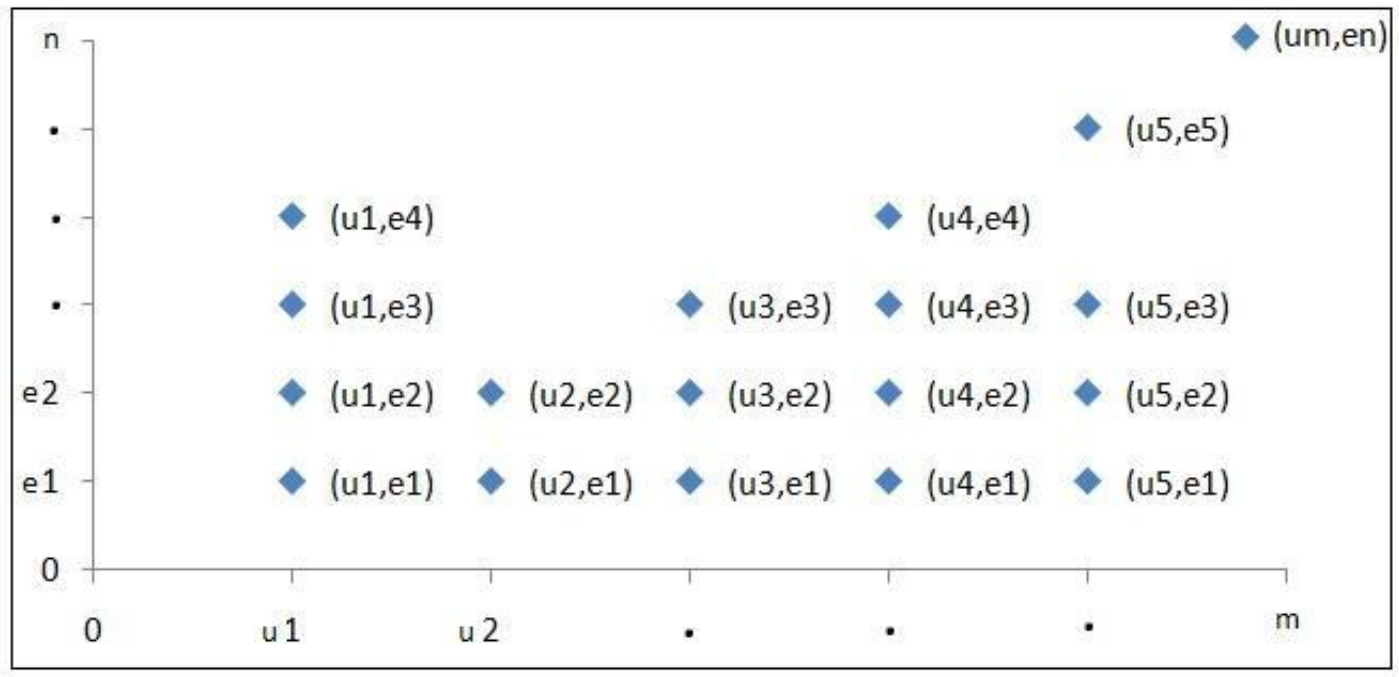

Fig. 3 Fuzzy soft sets 
Example 4: The fuzzy soft graph of example 2 defined in fuzzy soft set definitions are as:

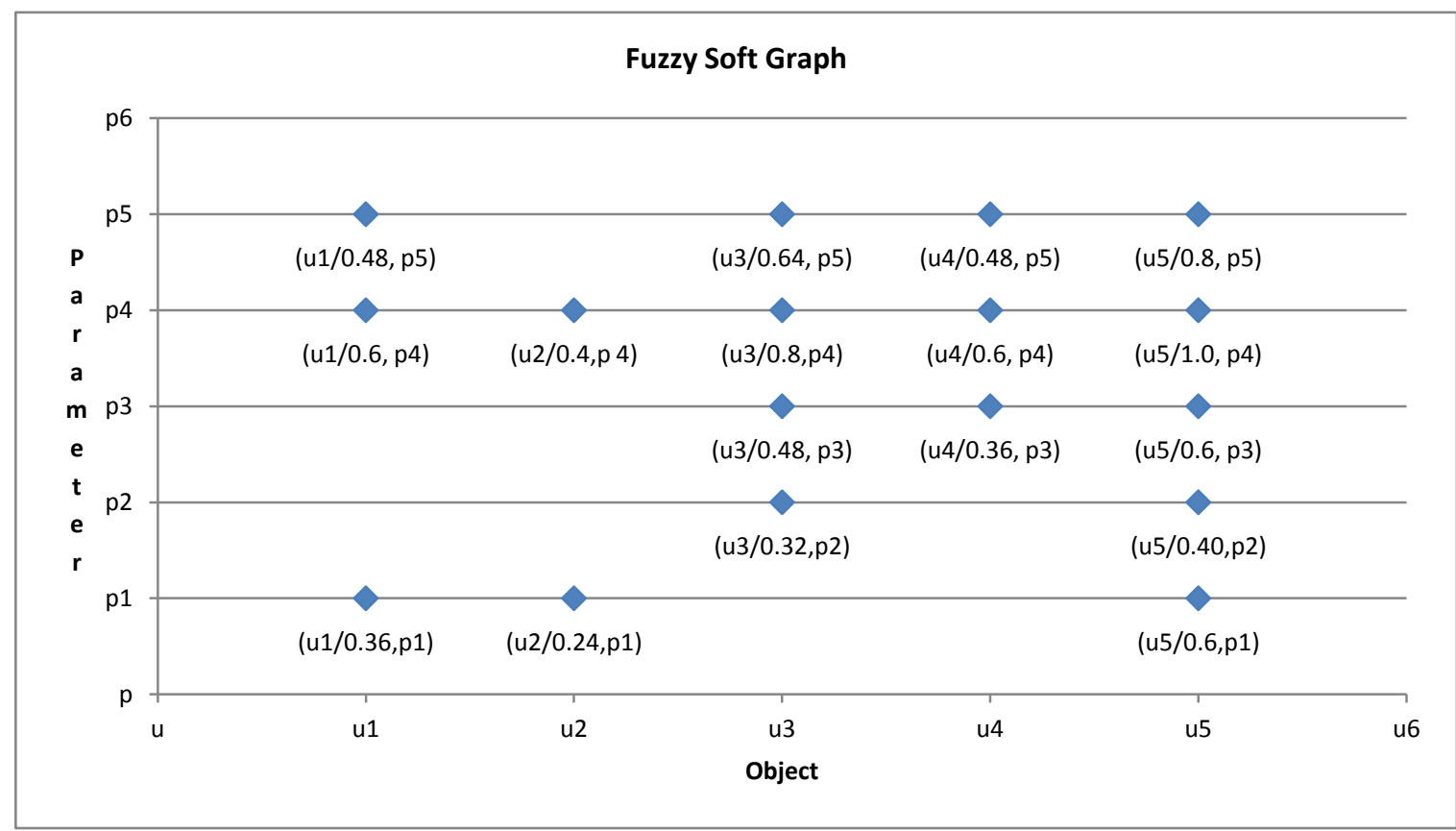

Fig. 4 Fuzzy Soft Graph of Example

\subsection{Proposed Procedure:}

The proposed step wise process of feature selection (data reduction) or decision making is as:

1. Consider a soft set table or fuzzy soft set table.

2. If a soft set table is considered then convert it into fuzzy soft set table by method discussed by Sharma et al. [19].

3. Draw a fuzzy soft graph by using fuzzy soft information tabled by using proposed technique.

4. Find the threshold value ' $T$ ' of the fuzzy soft information table by using formula $T=$ $\left(\sum_{i} \sum_{j} v_{i j}\right) /(|U| \times|P|)$, where $(|U| \times|P|)$ is the product of number of objects and parameters respectively and $v_{i j}$ is the entries in the fuzzy soft information table.

5. Represent the scattered points of graph into two form one for those whose values are less than or equal to threshold values and other for greater values.

6. Now two cases arise,

- In first case remove those scattered points representing less than or equal to threshold values. Now delete those objects not having any representation of

scattered points (after removal parameters).

- In second case remove those scattered points representing greater than threshold values. Now delete those parameters not having any representation of scattered points (after removal objects).

7. Now from the represented graph concluded the results of feature selection and decision making.

\section{APPLICATION IN FEATURE SELECTION AND DECISION MAKING}

Here same example is analyzed which is discussed by Maji et al. [4]. Let $U=\left\{h_{1}, h_{2}, h_{3}, h_{4}, h_{5}, h_{6}\right\}$ be a set of six houses, $\mathrm{E}=$ \{expensive, beautiful, wooden, cheap, in green surroundings, modern, in good repair, in bad repair\} be the set of parameters. Let $\mathrm{Mr}$. $\mathrm{X}$ is interested to buy a house on the following parameters subset $\mathrm{P}=\{$ beautiful, wooden, cheap, in green surroundings, in good repair $\}$. Let $\left\{p_{1}, p_{2}, p_{3}, p_{4}, p_{5}\right\}$ be symbolic representation of the set P. Soft set boolean-valued information system table specified by the soft set as in Table 4.1 .

Table 4.1 Soft Set Information Table

\begin{tabular}{|c|c|c|c|c|c|}
\hline $\boldsymbol{U} / \boldsymbol{P}$ & $\boldsymbol{p}_{\mathbf{1}}$ & $\boldsymbol{p}_{\mathbf{2}}$ & $\boldsymbol{p}_{\mathbf{3}}$ & $\boldsymbol{p}_{\mathbf{4}}$ & $\boldsymbol{p}_{\mathbf{5}}$ \\
\hline $\boldsymbol{h}_{\mathbf{1}}$ & 1 & 1 & 1 & 1 & 1 \\
\hline $\boldsymbol{h}_{\mathbf{2}}$ & 1 & 1 & 1 & 1 & 0 \\
\hline $\boldsymbol{h}_{\mathbf{3}}$ & 1 & 0 & 1 & 1 & 1 \\
\hline $\boldsymbol{h}_{\mathbf{4}}$ & 1 & 0 & 1 & 1 & 0 \\
\hline $\boldsymbol{h}_{\mathbf{5}}$ & 1 & 0 & 1 & 0 & 0 \\
\hline $\boldsymbol{h}_{\mathbf{6}}$ & 1 & 1 & 1 & 1 & 1 \\
\hline
\end{tabular}


Convert above soft set table into fuzzy soft set table by the method discussed by Sharma et al [19]. The fuzzy soft set table is as

Table 4.2 Fuzzy Soft Set Information Table

\begin{tabular}{|c|c|c|c|c|c|}
\hline $\boldsymbol{U} / \boldsymbol{P}$ & $\boldsymbol{p}_{\mathbf{1}}$ & $\boldsymbol{p}_{\mathbf{2}}$ & $\boldsymbol{p}_{\mathbf{3}}$ & $\boldsymbol{p}_{\mathbf{4}}$ & $\boldsymbol{p}_{\mathbf{5}}$ \\
\hline $\boldsymbol{h}_{\mathbf{1}}$ & 1.00 & 0.50 & 1.00 & 0.83 & 0.50 \\
\hline $\boldsymbol{h}_{\mathbf{2}}$ & 0.80 & 0.20 & 0.80 & 0.66 & 0 \\
\hline $\boldsymbol{h}_{\mathbf{3}}$ & 0.80 & 0 & 0.80 & 0.66 & 0.40 \\
\hline $\boldsymbol{h}_{\mathbf{4}}$ & 0.60 & 0 & 0.60 & 0.50 & 0 \\
\hline $\boldsymbol{h}_{\mathbf{5}}$ & 0.40 & 0 & 0.40 & 0 & 0 \\
\hline $\boldsymbol{h}_{\mathbf{6}}$ & 1.00 & 0.50 & 1.00 & 0.83 & 0.50 \\
\hline
\end{tabular}

The fuzzy soft graph representation of above fuzzy soft set table is shown below, in this graph $\left\{h_{1}, h_{2}, h_{3}, h_{4}, h_{5}, h_{6}\right\}$ is represented by $\left\{u_{1}, u_{2}, u_{3}, u_{4}, u_{5}, u_{6}\right\}$ respectively.

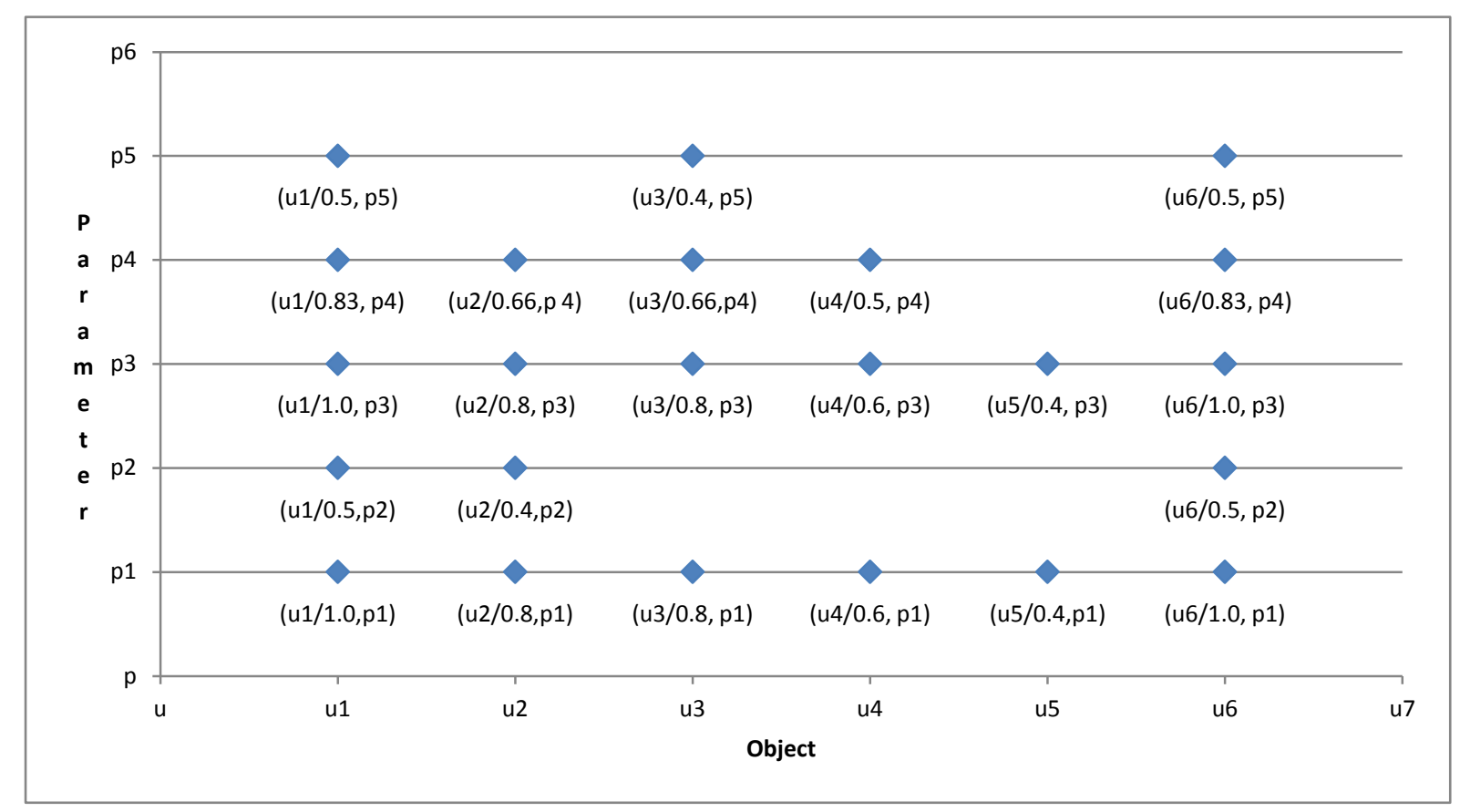

Fig. 5 Fuzzy Soft Graph of fuzzy soft information table

Now determine $T=\left(\sum_{i} \sum_{j} v_{i j}\right) /(|U| \times|P|)$ threshold value of fuzzy soft sets, thus by calculation the value of $\mathrm{T}=0.52$. Now represents scatter point in the fuzzy soft graph in two ways first is triangle for those values having less than or equal to threshold value and square for those value greater than threshold value. 


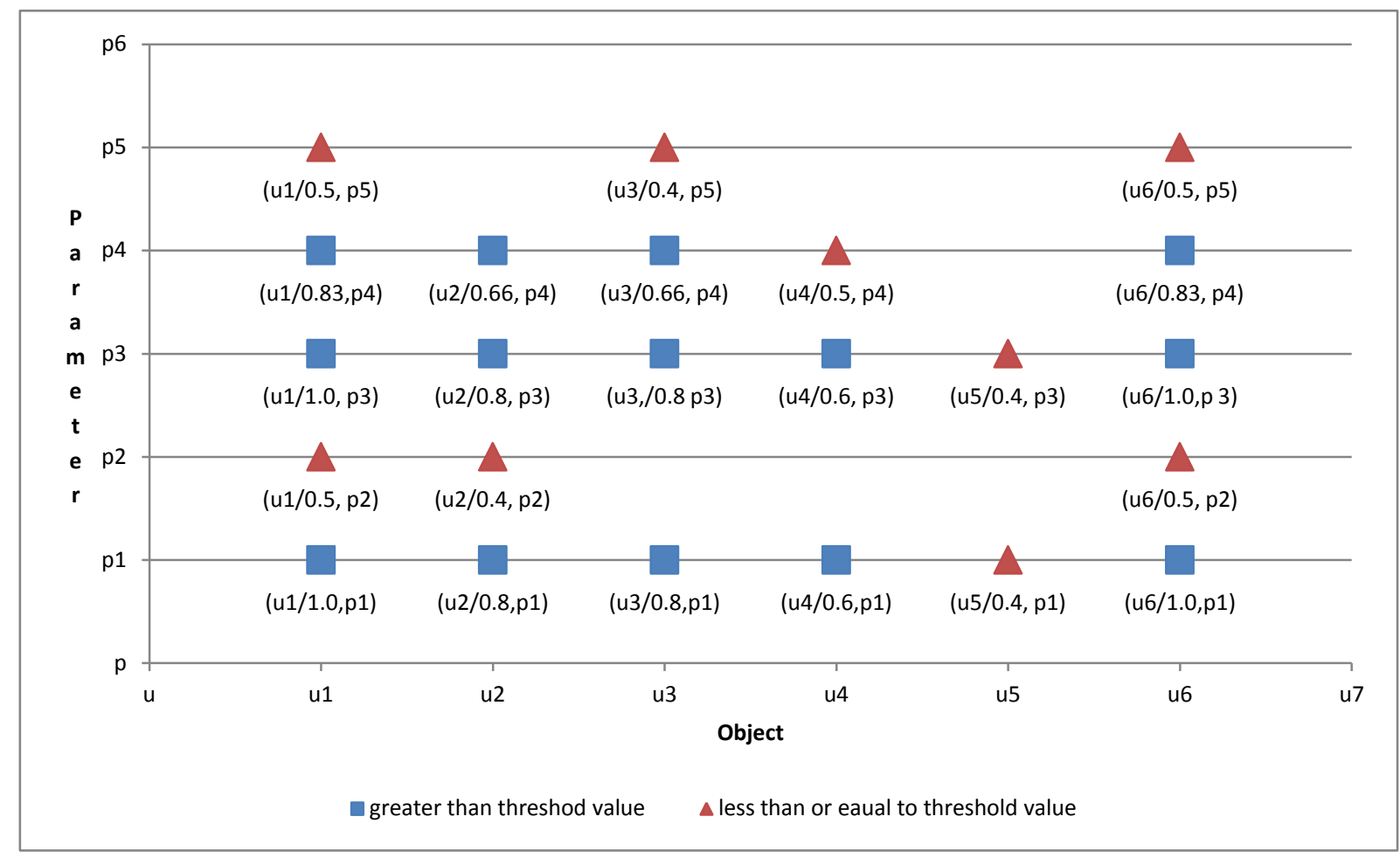

Fig. 6 Fuzzy Soft Graph of greater than and less than or equal to threshold value

Now here two cases arises in first case, from above graph delete those scatter points represented by triangles then fuzzy soft graph is represented as shown in fig. 7 and in second case remove those scattered point represented by square then fuzzy soft graph represented as shown in figure 8 .

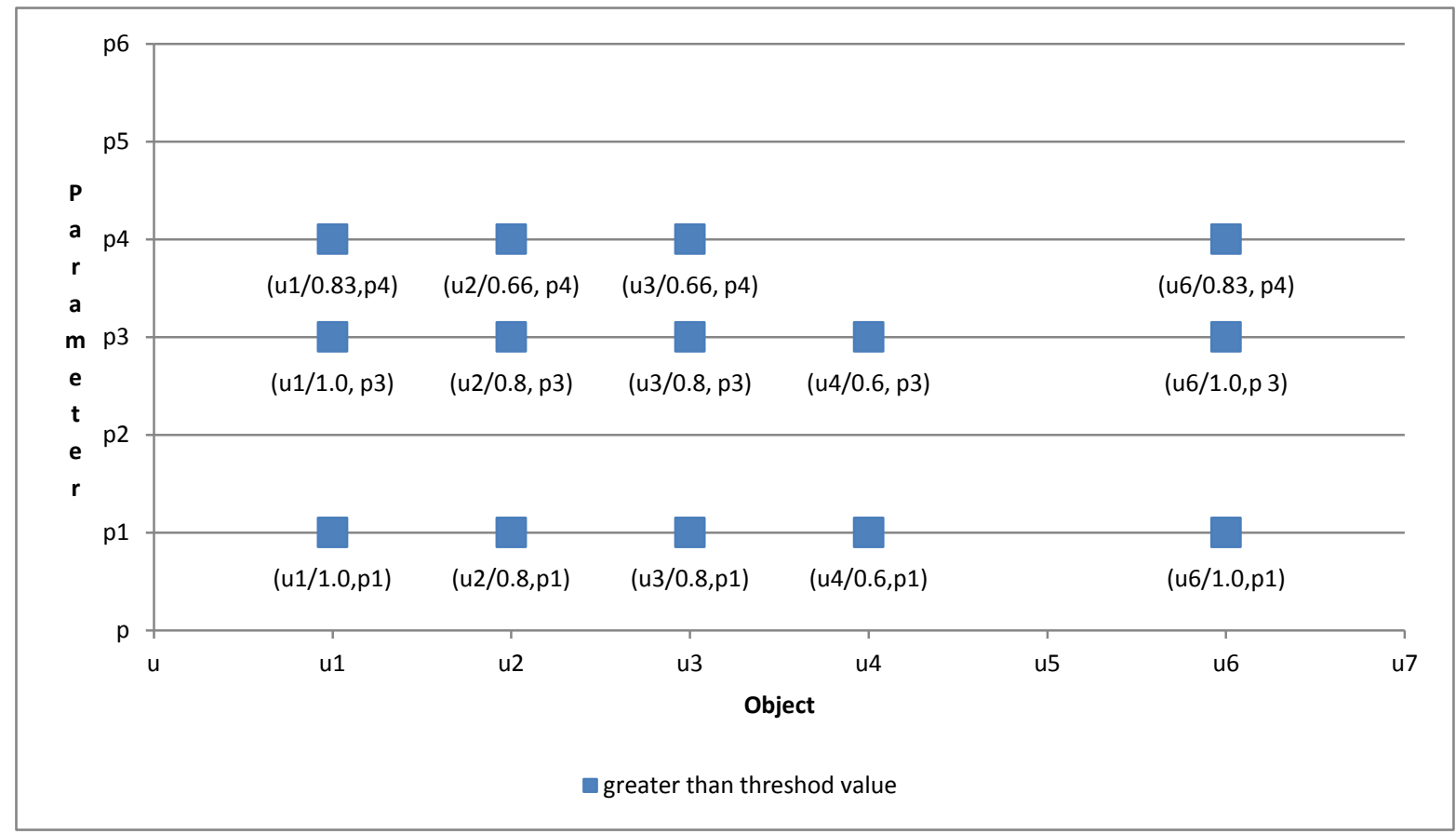

Fig. 7 Fuzzy Soft Graph represented values greater than threshold value (0.52) 


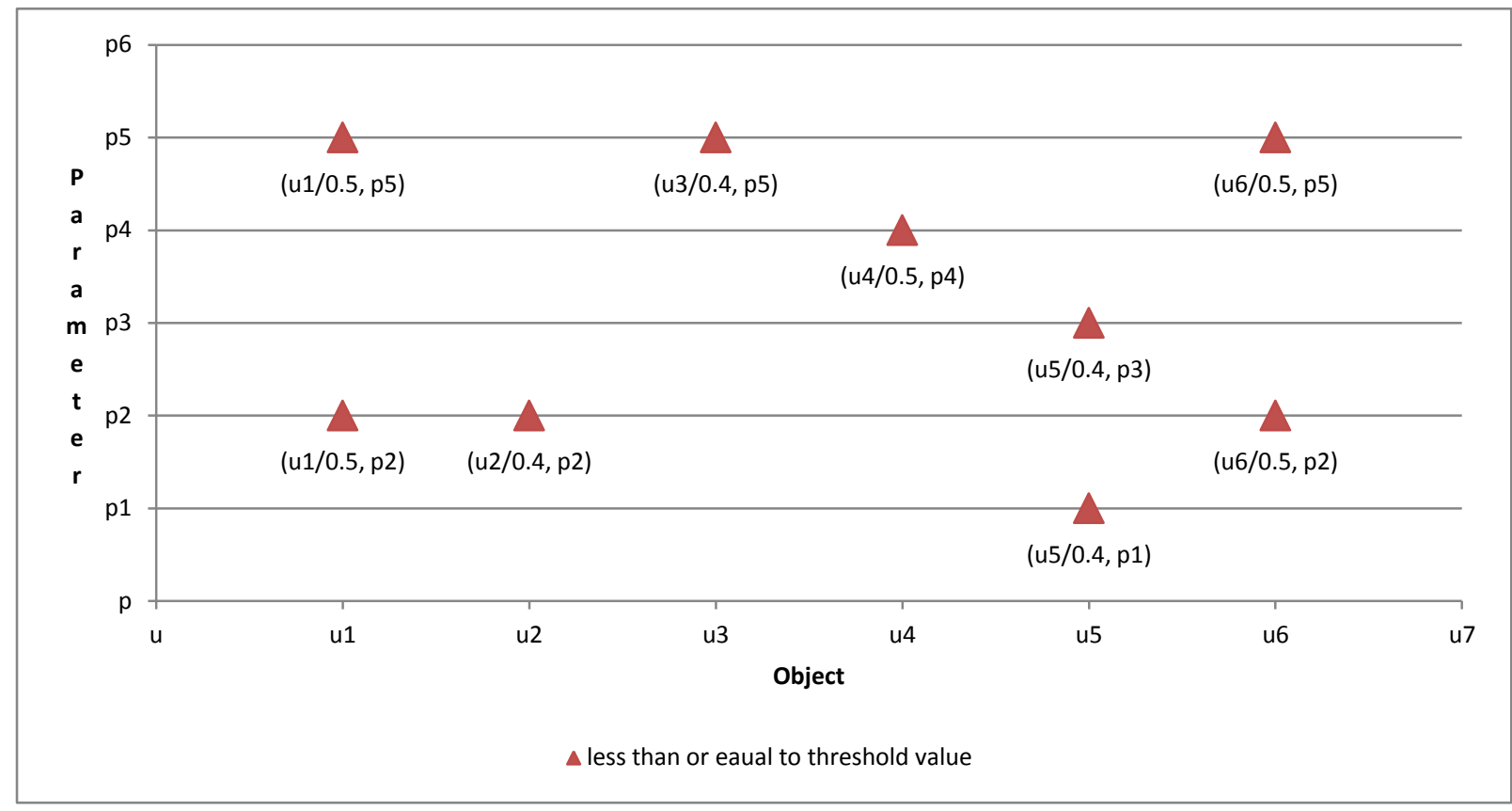

Fig. 8 Fuzzy Soft Graph values less than or equal to threshold value (0.52)

Now fig. 7 represent that there is no any scattered point with respect to $u_{5}$ or in other words that object $u_{5}$ does not show any relation to parameters. So delete the object $u_{5}$.
Now after removal of object $u_{5}$ in fig. 8 , fig. 8 is represented as fig. 9 (shown below) parameter $p_{1} \& p_{3}$ does not represent any scattered point so remove these parameters.

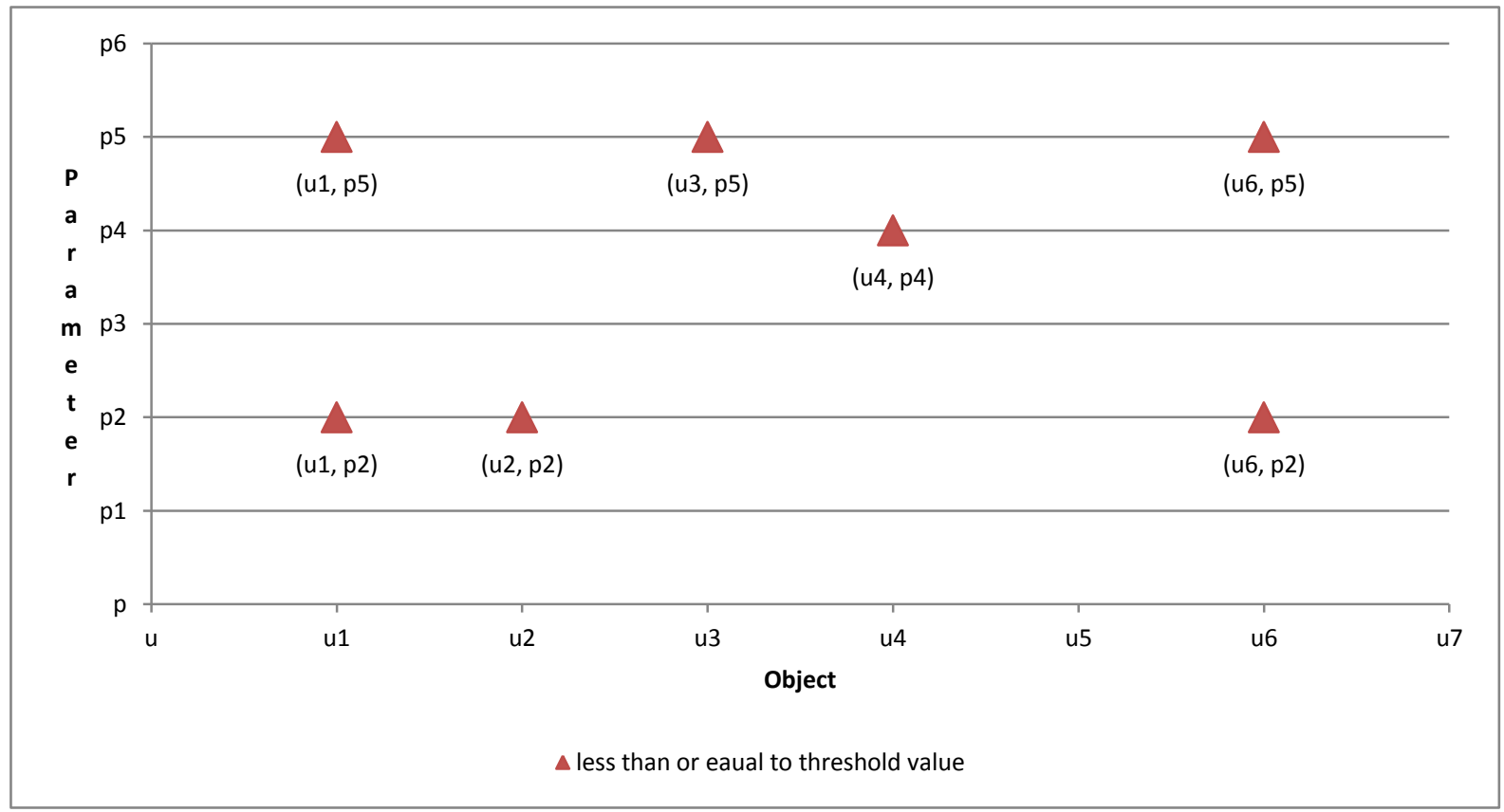

Fig. 9 Fuzzy Soft Graph after removal $u_{5}$

Now after removal of parameters and object fig. 9 , represent as fig. 10 graph shown below as: 


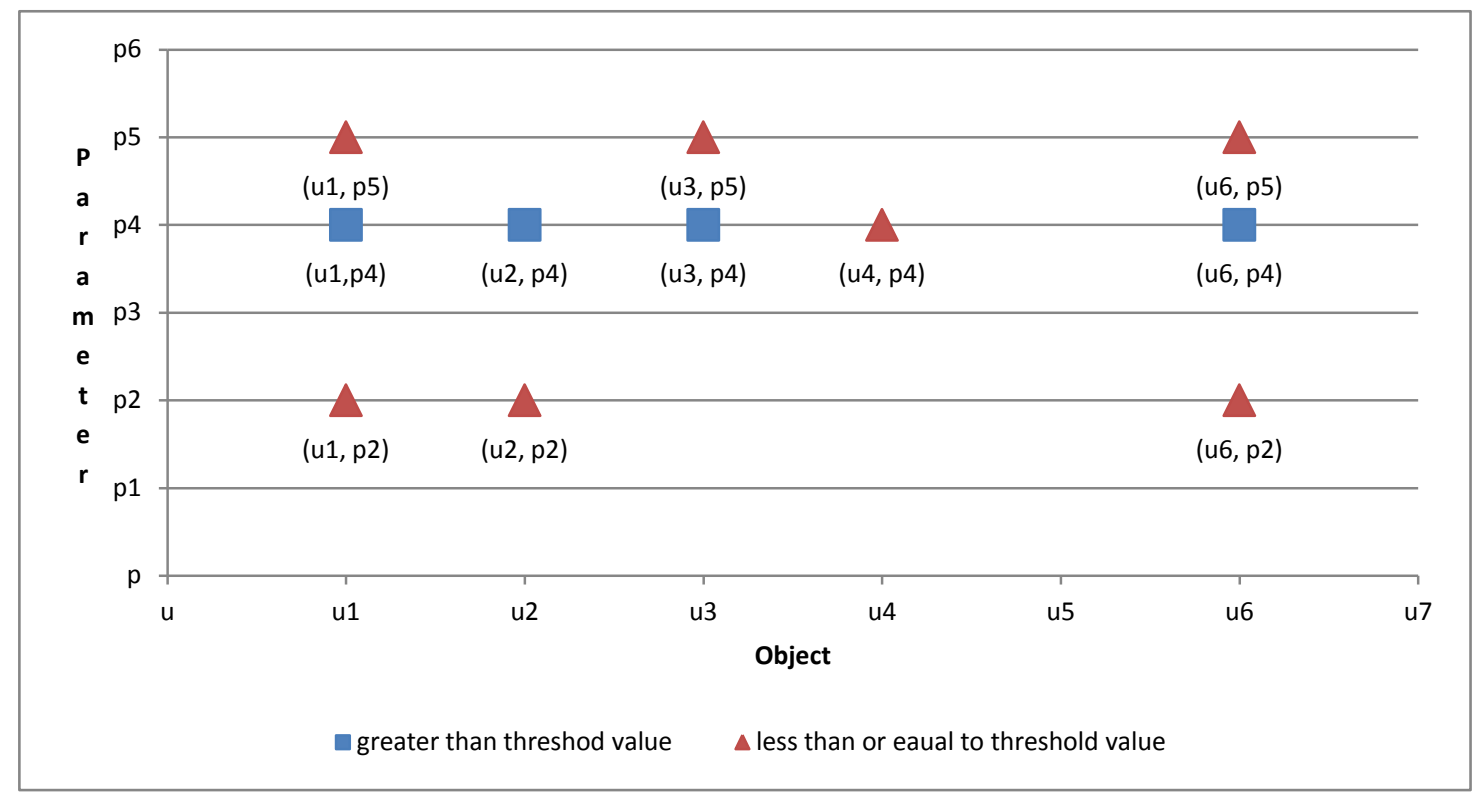

Fig. 10 Fuzzy Soft Graph after removal $p_{1}, p_{3} \& u_{5}$.

From figure 10 it is conclude that for given problem two parameters are reduced with-out affecting the Maji et al. [4] decisions. By using the information of parameter $p_{2}, p_{4} \& p_{5}$, house $h_{1} \& h_{6}$ are preffered

\section{CONCLUSIONS}

In this paper a fuzzy soft graph is describe with respect to soft graph described by Thomas et al. [12]. A method to solve decision making and feature selection problem by using fuzzy set theory and graph set theory via their hybridization are proposed. Finally an application of fuzzy soft graphs to decision making and feature selection is presented. In future this approach can be used in other application fields.

\section{REFERENCES}

[1] Zadeh, L. A. 1965. Fuzzy sets, Information and Control 8, 338-353, ().

[2] Molodtsov, D. 1999. Soft set theory- first results, Computers Math. Applic. 37 (4/5), 19-31.

[3] Maji, P. K., Roy, A. R. \& Biswas, R. 2001. Fuzzy soft sets, The Journal of fuzzy mathematics, ((3), 589-602.

[4] Maji, P. K., Roy, A. R. \& Biswas, R. 2002. An application of soft sets in a decision making Problem, Computer and Mathematics with application, 44, 10771083.

[5] Maji, P. K., Roy, A. R. \& Biswas, R. 2003. Soft set theory, Computers and Mathematics with Applications, $45,555-562$.

[6] Roy, A.R. \& Maji, P. K. 2007. A fuzzy soft set theoretic approach to decision making problems". J. Comput. Appl. Math., 203, 412-418.

[7] Chen, D., Tsang, E. C. C., Yeung, D. S. \& Wang, X. 2005. The parameterization reduction of soft sets and its applications, Computers and mathematics with applications, 49, 757-763.
[8] Gupta, P. \& Sharma, O. D. (2015). Feature Selection: An Overview, Impact: International Journal of Information Engineering and Technology, 1, 1- 12.

[9] Rosenfeld, A. 1975. Fuzzy graphs, Fuzzy sets and their applications, (L. A. Zadeh, K. S. Fu, M. Shimura, Eds) Academic Press, New York, 77-95.

[10] Thumbakara, R. K. \& George, B. 2014. Soft graphs, Gen. Math. Notes, vol. 21, no. 2, pp. 75-86, ICSRS Publication.

[11] Mohinta, S. \& Samanta, T. K. 2015. An introduction to fuzzy soft graph, MathematicaMoravica, vol. 19-2, 3548.

[12] Thomas, D. G. \& Arockiarani, I. 2013. Decision making via Soft graphs, International Journal of Mathematical Archive- 4(6), 50-55.

[13] Zou, Y. \& Xiao, Z. 2008. Data analysis approach of soft sets under incomplete information, Knowl. Base Syst., 21, 941-945.

[14] Yang, X., Lin, T. Y., Yang, J., Li, Y. and Yu, D. (2009). Combination of interval-valued fuzzy set and soft set, Comput. Math. Appl., 58, 521-527.

[15] Kong, Z., Gao, L., Wang, L. and Li, S. 2008. The normal parameter reduction of soft set and its algorithm, Comput. Math. Appl., 56, 3029-3037.

[16] Kong, Z., Gao, L. \& Wang, L. 2009. Comment On “ A fuzzy soft set theoretic approach to decision making problems", J. Comput. Appl. Math., 223, 540-542.

[17] Akram, M. \& Nawaz, S. 2015. On Fuzzy Soft Graphs, Italian Journal of Pure and Applied Mathematics, 34, 497-514, 9.

[18] Akram, M. \& Nawaz, S. 2015. Operatiom on Soft Graphs, Fuzzy information and Engineering, 7, 423-449.

[19] Sharma, O., Gupta, P. \& Abhishek, A novel approach of dimensionality reduction by fuzzy soft set (Communicated). 